International Journal of Pure and Applied Mathematics

Volume 87 No. $6 \quad 2013,863-870$

ISSN: $1311-8080$ (printed version); ISSN: 1314-3395 (on-line version)

url: http://www.ijpam.eu

doi: http://dx.doi.org/10.12732/ijpam.v87i6.15

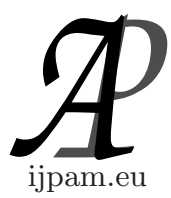

\title{
TOTAL BONDAGE NUMBER OF CERTAIN GRAPHS
}

\author{
Jasintha Quadras ${ }^{1}$, A. Sajiya Merlin Mahizl ${ }^{2}$ \\ ${ }^{1,2}$ Stella Maris College \\ Chennai, Tamilnadu, 600 086, INDIA
}

\begin{abstract}
A set $D$ of a vertices in a graph $G=(V, E)$ is said to be a total dominating set of $G$ if every vertex in $V$ is adjacent to some vertex in $D$. The total domination number $\gamma_{t}(G)$ is the minimum cardinality of a total dominating set. If $\gamma_{t}(G) \neq|V(G)|$, the minimum cardinality of a set $E_{0} \subseteq$ $E(G)$, such that $G-E_{0}$ contains no isolated vertices and $\gamma_{t}\left(G-E_{0}\right)>\gamma_{t}(G)$, is called the total bondage number of $G$. This paper determines the exact values of total bondage number of Wheel graph, Helm graph, Windmill graph, Circular necklace and Friendship graph.
\end{abstract}

AMS Subject Classification: 05C69

Key Words: total dominating set, total domination number, total bondage number

\section{Introduction}

The total domination in graphs was introduced by Cockayne et al.[3] in 1980. The total domination in graphs has been extensively studied in the literature. In 2009, Henning [7] gave a survey of selected recent results on this topic.

In 1990, Fink et al.[4] introduced the bondage number as a parameter for measuring the vulnerability of the interconnection network under link failure. The minimum dominating set of sites plays an important role in the network for it dominates the whole network with the minimum cost. So we must consider whether its function remains good under attack. Suppose that someone such as

Received: September 6, 2013

(c) 2013 Academic Publications, Ltd. url: www.acadpubl.eu 
a saboteur does not know which sites in the network take part in the dominating role, but does know that the set of these special sites corresponds to a minimum dominating set in the related graphs. Then how many links does he have to attack so that the cost cannot remain the same in order to dominate the whole network? That minimum number links is just the bondage number.

We consider only simple finite graphs without isolated vertices. For graph theoretic terminology we refer to Bondy and Murty[2]. To each vertex $v$ of a graph $G, N(v)$ denotes the set of all vertices of $G$ which are adjacent to $v$. For any subset $S \subseteq V, N(S)=\cup\{N(v) \mid v \in S\}$.

A set $D$ of vertices in a graph $G$ is said to be a dominating set if every vertex in $V-D$ is adjacent to some vertex in $D$. We call $D$ a total dominating set for $G$ if every vertex in $V$ is adjacent to some vertex in $D$.(i.e., $N(D)=V$ ). The minimum cardinality of a dominating set (a total dominating set) of $G$ is denoted by $\gamma(G),\left(\gamma_{t}(G)\right)$ and is called the domination number (the total domination number) of $G$. It is clear that $\gamma(G) \leq \gamma_{t}(G) \leq 2 \gamma(G)$ for any graph $G$ without isolated vertices.

The bondage number $b(G)$ of a nonempty graph $G$ is the minimum number of edges whose removal from $G$ results in a graph with larger domination number than $\gamma(G)$. Kulli and Patwari[9] proposed the concept of the total bondage number for a graph. The total bondage number $b_{t}(G)$ of a graph $G$ is the minimum number of edges whose removal results in a graph with total domination number larger than $\gamma_{t}(G)$. In [6], $\mathrm{Hu}$ and $\mathrm{Xu}$ showed that the problem of determining total bondage number for general graphs is NP-hard.

Kulli and Patwari[9] calculated the exact values of $b_{t}(G)$ for some standard graphs such as a cycle $C_{n}$ and a path $P_{n}$ for $n \geq 4$, a complete bipartite graph $K_{m, n}$ and a complete graph $K_{n}$. Sridharan et al.[12] showed that for any positive integer $k$ there exists a tree $T$ with $b_{t}(T)=k$. Fu-Tao et al. [5] determined the exact values of grid graphs $b_{t}\left(G_{n, 2}\right), b_{t}\left(G_{n, 3}\right)$ and established some upper bounds of $b_{t}\left(G_{n, 4}\right)$.

Nader Jafari Rad et al.[10] improved the upper bound for the total bondage number of a tree and showed an interesting constructive characterization of a certain class of trees achieving equality for the upper bound. Further they derived some upper bounds on $b_{t}(G)$ in terms of maximum and minimum degrees, which in particular improve similar previous bounds. Jianxiang Cao et al.[8] computed the total bondage number of generalized peterson graphs.

In this paper, we determine the exact values of total bondage number of Wheel graph, Helm graph, Windmill graph, Circular necklace and Friendship graph. 


\section{Total Bondage Number}

Definition 2.1. Let $G$ be a graph. If there exists $E_{0} \subset E(G)$ such that (i) there is no isolated vertex in $G-E_{0}$ and (ii) $\gamma_{t}\left(G-E_{0}\right)>\gamma_{t}(G)$, then the edge set $E_{0}$ is called a total bondage edge set for $G$. If there is at least one total bondage edge set for $G$, we define $b_{t}(G)=\min \left\{\left|E_{0}\right|: E_{0}\right.$ is a total bondage edge set of $G$ \}. Otherwise we put $b_{t}(G)=\infty$. For star graph $K_{1, n}$, we define $b_{t}(G)=\infty$.

\subsection{Wheel Graph}

A wheel graph $W_{n}$ of order $n$ is a graph that contains an outer cycle of order $n-1$, and for which every vertex in the cycle is connected to one other vertex (which is known as the hub)[1]. The edges of a wheel which include the hub are called spokes. See Figure 1. This plays an important role in the circuit layout and interconnection network designs. In other words, a wheel graph $W_{n}$ is obtained when a additional vertex $u$ not on $C_{n-1}$ is joined to each of the $n-1$ vertices namely $v_{1}, v_{2}, \ldots . v_{n-1}$ in $C_{n-1}$ by new edges. See Figure 1 .

Lemma 2.1. Let $G$ be a wheel graph. Then $\gamma_{t}(G)=2$

Theorem 2.1. If $W_{n}$ is a wheel graph with $n>4$, then $b_{t}\left(W_{n}\right)=2$.

Proof. $S=\left\{u, v_{i}\right\}, 1 \leq i \leq n-1$ is clearly a minimum total dominating set of $G$ by lemma 2.1 .

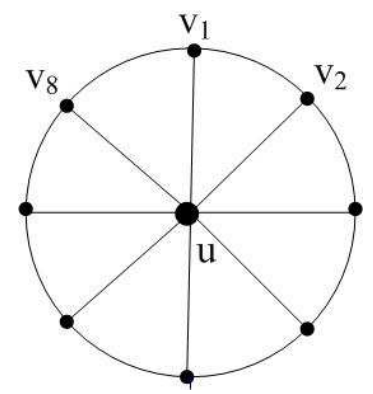

Figure 1 Wheel graph $W_{9}$.

Even if we remove any edge from the outer cycle, $S$ is a minimum total dominating set of $G$. Further, when we remove any spoke edge, the total domination number remains the same. Thus, $b_{t}(G) \geq 2$. Removal of two adjacent spoke edges namely $u v_{i}, u v_{i+1}, S=\left\{u, v_{i-1}, v_{i+2}\right\}, 1 \leq i \leq n-1$ is a minimum total dominating set and increases the total domination number to 3. Hence, $b_{t}(G)=2$. 


\subsection{Helm Graph}

A Helm graph is denoted by $H_{n}$ is a graph obtained by attaching a single edge and vertex to each vertex of the outer circuit of a wheel graph $W_{n}$. The number of vertices of $H_{n}$ is $2 n-1$ and the number of edges $3(n-1)$.

Lemma 2.2. Let $G$ be a helm graph. Then $\gamma_{t}(G)=n-1$

Proof. By the definition of a helm graph, there are $n-1$ pendent vertices. To totally dominate all these vertices, we need $n-1$ vertices which are adjacent to pendent vertices. From Figure 2 , the vertices $\left\{v_{1}, v_{2}, \ldots \ldots v_{n-1}\right\}$ form a minimum total dominating set of $G$. Thus, $\gamma_{t}(G)=n-1$.

Theorem 2.2. If $H_{n}$ is a helm graph, then $b_{t}\left(H_{n}\right)=2$.

Proof. $S=\left\{v_{1}, v_{2}, \ldots \ldots \ldots, v_{n-1}\right\}$ is clearly a minimum total dominating set of $G$ by lemma 2.2 .

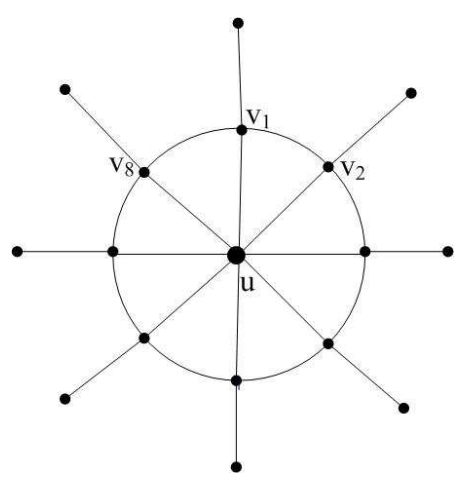

Figure 2 Helm graph $H_{9}$.

Even if we remove any edge from the outer cycle, $S$ is a minimum total dominating set of $G$. Further, when we remove any spoke edge, the total domination number remains the same. Thus, $b_{t}(G) \geq 2$. Removal of any two adjacent edges in $C_{n-1}$, increases the total domination number to $n$. Hence, $b_{t}(G)=2$.

\subsection{Windmill Graph}

The windmill graph $W d(k, n)$ can be constructed by joining $n$ copies of the complete graph $K_{k}$ with a common vertex. It has $(k-1) n+1$ vertices and $n k(k-1) / 2$ edges[11]. 
Lemma 2.3. [5] If $K_{k}$ is the complete graph with $k \geq 5$ vertices, then

$$
b_{t}\left(K_{k}\right)=2 k-5 .
$$

The following result is easy to prove.

Lemma 2.4. If $W d(k, n)$ is a windmill graph with $k \geq 5$, then

$$
\gamma_{t}(W d(k, n))=2 .
$$

Theorem 2.3. If $W d(k, n)$ is a windmill graph with $k \geq 5$, then

$$
b_{t}(W d(k, n))=2 k-5 .
$$

Proof. From Lemma 2.3 and the definition of windmill graph, the total bondage number of one copy of complete graph is the total bondage number of windmill graph. Hence, $b_{t}(W d(k, n))=2 k-5, k \geq 5$.

\subsection{Circular Necklace}

Let $K_{m}$ and $K_{t_{i}}$ be complete graphs on $m$ (say $v_{1}, v_{2}, \ldots \ldots v_{m}$ ) and $t_{i}$ vertices respectively. Let $t_{i}=2^{r_{i}}, 1 \leq i \leq m$, and $r_{1}=r_{2}, r_{i+1}=r_{i}+1$ for all $2 \leq i \leq m-1$ such that $K_{m} \biguplus K_{t_{i}}$ has just $v_{i}$ as a cut vertex, where $r_{i}$ is an integer and $1 \leq i \leq m$. The resultant graph $K_{m} \biguplus\left[\bigcup_{i=1}^{m} K_{t_{i}}\right]$ is a circular necklace denoted by $C N\left(K_{m} ; K_{t_{1}}, K_{t_{2}}, \ldots . . K_{t_{m}}\right)[11]$. See Figure 3.

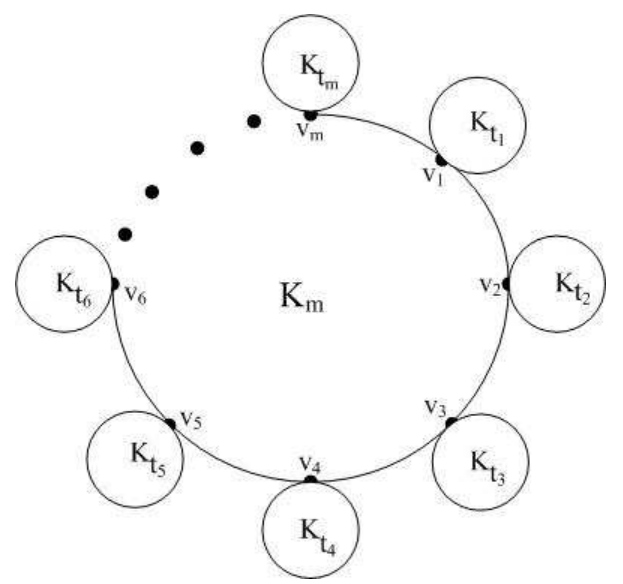

Figure 3 Circular necklace $C N\left(K_{m} ; K_{t_{1}}, K_{t_{2}}, \ldots . . K_{t_{m}}\right)$.

Lemma 2.5. Let $G$ be a circular necklace graph. Then $\gamma_{t}(G)=m$ 
Proof. From the definition of circular necklace graph, both $K_{m}$ and $K_{t_{i}}$ are complete graphs. Since any one vertex dominates all the remaining vertices in the complete graph, any one vertex from each complete graph contained in a minimum total dominating set of $G$. From Figure 3 , the vertices $\left(v_{1}, v_{2}, \ldots \ldots v_{m}\right)$ form a minimum total dominating set of $G$. Thus, $\gamma_{t}(G)=m$.

Theorem 2.4. Let $G$ be a circular necklace graph. Then $b_{t}(G)=1$

Proof. From lemma 2.5, $\gamma_{t}\left(C N\left(K_{m} ; K_{t_{1}}, K_{t_{2}}, \ldots . K_{t_{m}}\right)=m\right.$.

Removal of an edge incident at exactly one $v_{i}$, increases the total domination number to $m+1$. Hence, $b_{t}(G)=1$.

\subsection{Friendship Graph}

The friendship graph $F_{n}$ can be constructed by joining $n$ copies of the cycle graph $C_{3}$ with a common vertex.

Lemma 2.6. Let $G$ be friendship graph. Then $\gamma_{t}(G)=2$.

Theorem 2.5. If $F_{n}$ is a friendship graph, then $b_{t}\left(F_{n}\right)=2$.

Proof. $S=\left\{u, v_{i}^{j}\right\}, 1 \leq i \leq n$ and $1 \leq j \leq n$ is clearly a minimum total dominating set of $G$ by lemma 2.6.

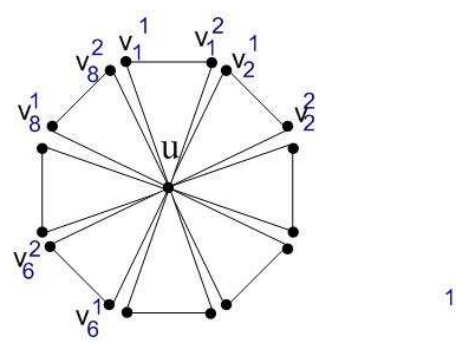

Figure 4 Friendship graph $F_{8}$.

Even if we remove an edge not incident with $u, S$ is a minimum total dominating set of $G$. Further, when we remove any spoke edge, the total domination number remains the same. Thus, $b_{t}\left(F_{n}\right) \geq 2$. Removal of any two spoke edges, increases the total domination number to 3 . Hence, $b_{t}\left(F_{n}\right)=2$. .

\section{Conclusion}

In this paper we determine the exact values of total bondage number of a wheel graph, helm graph, windmill graph, circular necklace, friendship graph. The 
problem of finding the total bondage number of architecture such as Pyramid networks, Circulant networks are under investigation.

\section{References}

[1] M. Arockiaraj, P.Manuel, I.Rajasingh and B.Rajan, Wirelength of 1-fault hamiltonian graphs into wheels and fans. Information Processing Letters, 111, (2011), 921-925.

[2] J.A.Bondy,U.S.R.Murty, Graph Theory with Applications. Macmillan (1976).

[3] E.J. Cockayne, R.M. Dawes, S.T. Hedetniemi. Total domination in graphs. Networks, 10, (1980), 211-219.

[4] J. F. Fink, M. S. Jacobson, L. F. Kinch and J. Roberts, The bondage number of a graph. Discrete Math., 86, (1990), 47-57.

[5] Fu-Tao Hu, You Lu, Jun-Ming Xu, The total bondage number of grid graphs. arXiv:1109.3929v1 [math.CO], (2011).

[6] Fu-Tao Hu and J.-M. Xu, Complexity of Bondage and Reinforcement. A manuscript submitted to Discrete Applied Mathematics, (2010).

[7] M. A. Henning, A survey of selected recent results on total domination in graphs. Discrete Mathematics, 309(1) (2009), 32-63.

[8] Jianxiang Cao, Minyong Shi, Bin Wu, Research on the Total Bondage Number of a Spectial Network. Third International Joint Conference on Computational Science and Optimization, 2, (2010), 456-458.

[9] V. R. Kulli and D. K. Patwari, The total bondage number of a graph. Advances in Graph Theory, Vishwa International Publications, (1991), 227235.

[10] Nader Jafari Rad, Joanna Raczek, Some Progress on Total Bondage in Graphs. Graphs and Combinatorics, (2013)

[11] I.Rajasingh, B.Rajan, R. Sundara Rajan, Embedding of hypercubes into necklace, windmill and snake graph. Information Processing Letters, 112, (2012), 509-515. 
[12] N. Sridharan, M. D. Elias, V. S. A. Subramanian, Total bondage number of a graph. AKCE Int. J. Graphs Combin, 4(2), (2007), 203-209. 